\title{
Valproic Acid Treatment for Spinal Muscular Atrophy Patient with Abnormal Electroencephalogram Findings
}

Toshio Saito $^{1 *}$, Indra Sari Kusuma Harahap ${ }^{2}$, Harutoshi Fujimura1, Kayoko Saito ${ }^{3}$ and Hisahide Nishio ${ }^{2}$

${ }^{1}$ Department of Neurology, Division of Child Neurology, Toneyama National Hospital, Toneyama, Toyonaka, Japan

${ }^{2}$ Department of Community Medicine and Social Healthcare Science, Kobe University Graduate School of Medicine, Kusunoki-Cho, Chuo-Ku, Kobe, Japan

3Institute of Medical Genetics, Tokyo Women's Medical University, Kawada-cho, Shinjuku-ku, Tokyo, Japan

\begin{abstract}
Background: Valproic Acid (VPA), an anticonvulsant, is a histone deacetylase inhibitor that can increase the Survival Motor Neuron (SMN) level in Spinal Muscular Atrophy (SMA) patients and anticipated to be an effective therapeutic agent for SMA.

Patient description: VPA was administered for SMA to a 6-year-old female who showed non-symptomatic abnormal electroencephalogram findings, as well as SMA signs and symptoms. Although the VPA concentration of our patient remained in a low range, electroencephalogram abnormalities disappeared within 10 months after starting administration. On the other hand, clinical signs and symptoms of SMA, respiratory function values, and modified Hammersmith Functional Motor Scale results showed no remarkable changes. We also analyzed survival motor neuron $(S M N)$ transcripts and splicing factor transcripts, such as splicing factor 2/alternative splicing factor (SF2/ASF) and heterogeneous nuclear ribonucleoprotein A1 (hnRNPA1), using quantitative real-time PCR assays performed before and after VPA treatment. The amounts of the FL-SMN, total-SMN, ASF/SF2, and hnRNPA1 transcripts showed an increasing tendency, whereas that of $\triangle 7-S M N$ was decreased.
\end{abstract}

Conclusion: Our findings indicate a discrepancy between SMN transcript response, and improvements in SMA symptoms and signs following VPA administration in some SMA patients.

Keywords: Heterogeneous nuclear ribonucleoprotein A1 (hnRNPA1); Spinal muscular atrophy (SMA); Splicing factor 2/ alternative splicing factor (SF2/ASF); Survival motor neuron $(S M N)$; Valproic acid (VPA)

\section{Introduction}

Spinal muscular atrophy (SMA) is a common autosomal recessive neuromuscular disorder characterized by progressive muscular atrophy, as well as weakness of the limbs and trunk derived from degeneration of motor neurons of the spinal cord. The disease is classified into 5 groups; type 0 (prenatal), type 1 (Werdnig-Hoffman disease), type 2 (Dubowitz disease), type 3 (Kugelberg-Welander disease), and type 4 (adult form), based on age at onset and achievement of motor milestones [1].

The survival motor neuron $(S M N)$ gene is responsible for SMA. Two highly homologous copies of that gene, namely SMN1 and SMN2, exist within the SMA genetic region on chromosome 5q11.2-13.3 [2]. SMN1 is homozygously deleted or interrupted in more than $95 \%$ of SMA patients, which results in a deficiency of the $S M N$ protein $[2,3]$. SMN1 and SMN2 encode the same protein, because of a synonymous nucleotide change, and their difference is only a single nucleotide change, as nucleotide +6 in the coding region of exon 7 in SMN1 is $\mathrm{C}$ and that of SMN2 is T. However, they also show different splicing patterns [4]. All SMN1-derived transcripts contain exon 7, namely, the full-length $S M N$ (FL-SMN) transcript, while the majority of $S M N 2$-derived transcripts lack exon $7(\triangle 7-S M N)$, because the $\mathrm{C}$ to T change in $S M N 2$ at nucleotide position +6 in exon 7 induces exon skipping [4]. This genetic mechanism is enhanced by an exonic splicing enhancer (ESE) and exonic splicing silencer (ESS). In SMN1, a heptamer sequence motif including $C$ at nucleotide position +6 in exon 7 shapes a splicing factor 2/alternative splicing factor (SF2/ASF) binding site as an ESE, resulting in exon 7 inclusion [5]. On the other hand, the corresponding T nucleotide in SMN2 disrupts the ESE motif and shapes a heterogeneous nuclear ribonucleoprotein A1 (hnRNPA1) binding site as an ESS, resulting in exclusion of exon 7 [6].
The SMN2 copy number is inversely correlated with disease severity. Although SMN2 does not fully compensate for loss or dysfunction of $S M N 1$, a higher $S M N 2$ copy number ameliorates the clinical phenotype [1,7]. SMN2 is thought to compensate for loss of SMN1 by producing a small amount of full-length $S M N$ protein. Thus, treatment strategies for SMA have focused on increasing the expression of SMN2. Valproic acid (VPA) is an anticonvulsant used for treatment of epileptic patients. In addition, the agent is also a histone deacetylase (HDAC) inhibitor that can increase $S M N$ levels in SMA patients via activation of SMN2 transcription and splicing correction of SMN2 exon $7[8,9]$. Thus, VPA is anticipated to be an effective therapeutic drug for SMA [10-12]. Here, we report VPA treatment given to a 6-year-old girl with SMA for electroencephalogram abnormalities. We evaluated changes in electroencephalogram findings, as well as SMA symptoms and signs, and also the SMN transcript, ASF-SF2, and hnRNPA1 before and after starting VPA treatment.

\section{Case Report}

A 6-year-old girl with SMA came to our hospital for evaluations of respiratory function and general condition. Developmental milestones were delayed, and the patient showed unstable sitting and walking, though mental development was normal. Previous

*Corresponding author: Toshio Saito, Department of Neurology, Division of Child Neurology, Toneyama National Hospital, 5-1-1 Toneyama, Toyonaka, Japan, Tel: 81-6-6853-2001; E-mail: saitot@toneyama.go.jp

Received September 15, 2017; Accepted November 23, 2017; Published November 27, 2017

Citation: Saito T, Harahap ISK, Fujimura H, Saito K, Nishio H (2017) Valproic Acid Treatment for Spinal Muscular Atrophy Patient with Abnormal Electroencephalogram Findings. J Neurol Disord 5: 367. doi:10.4172/2329-6895.1000367

Copyright: ( 2017 Saito T, et al. This is an open-access article distributed under the terms of the Creative Commons Attribution License, which permits unrestricted use, distribution, and reproduction in any medium, provided the original author and source are credited. 
Citation: Saito T, Harahap ISK, Fujimura H, Saito K, Nishio H (2017) Valproic Acid Treatment for Spinal Muscular Atrophy Patient with Abnormal Electroencephalogram Findings. J Neurol Disord 5: 367. doi: 10.4172/2329-6895.1000367

Page 2 of 4

findings for the patient showed SMN1 deletion, and that she carried 3 copies of SMN2 and the neuronal apoptosis inhibitory protein gene (NAIP). Non-invasive ventilation (NIV) was used during sleep at night. Electroencephalogram findings revealed abnormal spikes and waves, with sharp waves frequently occurring in the right temporal and occipital regions, though related clinical symptoms were not apparent (Figure 1). Brain magnetic resonance imaging (MRI) showed no abnormalities (Figure 2). We determined that treatment for the abnormal electroencephalogram findings would be the first objective, thus VPA administration was decided. In addition, we hoped to evaluate its effects on laboratory findings, as well as SMA symptoms and signs.

\section{Ethical Approval}

Informed consent was obtained from the patient and her family prior to beginning the study, and the protocol was approved by the local ethical committees of Toneyama National Hospital and Kobe University.

\section{Clinical Course of Patient}

VPA was started at $25 \mathrm{mg} /$ day and then gradually increased to 225 $\mathrm{mg}$ /day over a period of 10 months. L-carnitine was also administered at $100 \mathrm{mg} /$ day from 5 months after starting VPA treatment. Following initiation of VPA therapy, abnormal waves shown in electroencephalogram findings disappeared. On the other hand, the modified Hammersmith Functional Motor Scale for SMA (MHFMS) before starting VPA was $1 / 40$ and remained at $1 / 40$ after 10 months of VPA treatment, indicating no remarkable change (Figure 3).

For respiratory function, we assessed vital capacity (VC), maximum insufflation capacity (MIC), and cough peak flow (CPF) [13]. VC before starting VPA administration was in a range of $610-700 \mathrm{ml}$, while that was $530-700 \mathrm{ml}$ after starting treatment, indicating a decrease in $\mathrm{VC}$ after starting VPA. Furthermore, MIC before treatment was $850 \mathrm{ml}$, then slightly decreased to $700 \mathrm{ml}$ after 10 months, while CPF before treatment was $50 \mathrm{l} /$ minute and slightly increased to $70 \mathrm{l} /$ minute after 10 months. VPA concentration was $11-16 \mu \mathrm{g} / \mathrm{ml}$. There were no abnormal changes in liver function and carnitine fraction also remained normal.

\section{SMN, SF2/ASF, and hnRNPA1 transcript}

We analyzed peripheral white blood cell samples obtained from the patient prior to initiating VPA treatment and at 7 months after beginning treatment ( $125 \mathrm{mg} /$ day). SMN transcripts (FL-SMN, total-SMN, $\Delta 7-$ $S M N), S F 2 / A S F$, and hnRNPA1 were examined using quantitative realtime PCR (qRT-PCR) analysis, with the glyceraldehyde-3-phosphate dehydrogenase (GAPDH) transcript as the internal reference, with the levels of SMN, SF2/ASF, and hnRNPA1 expressed relative to that of GAPDH [14]. Details of the methods utilized for qRT-PCR have been reported [14].

The FL-SMN transcript amount showed an increasing tendency after starting VPA treatment, with the value after 7 months reaching a relative ratio of 0.87 , an increase of 0.18 points from the pre-treatment value. That also reached $87 \%$ of the values for the FL-SMN transcript in a healthy control (Figure 4). After starting VPA treatment, the $\triangle 7-S M N$ transcript amount showed a decreasing tendency, while the amount of total-SMN transcript showed a slight increasing tendency. Furthermore, the SF2/ASF transcript amount showed an approximately 2-fold increase after 7 months as compared to the pretreatment value, while the hnRNPA1 transcript showed a very slight increase at that time point.

\section{Discussion}

In the present patient, electroencephalogram abnormalities disappeared after starting VPA administration at a low concentration, whereas motor function did not change and respiratory function gradually deteriorated. Thus, there was no evident efficacy of administration of a low concentration of VPA in regard to motor

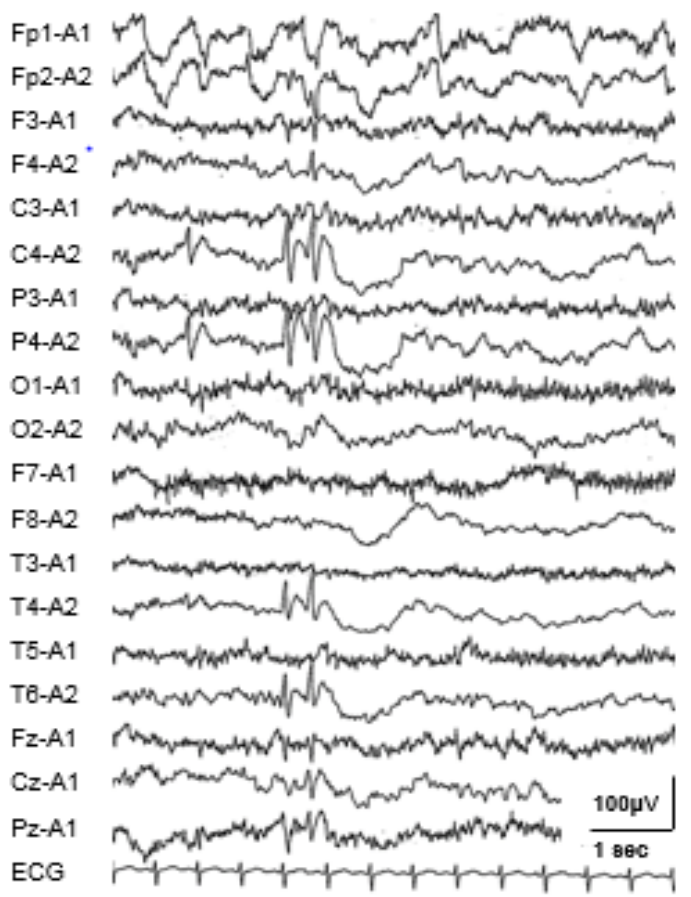

Figure 1: Electroencephalogram obtained before starting VPA treatment. Abnormal spikes and waves, including sharp waves frequently occurred in the right temporal and occipital regions, though clinical symptoms were not apparent valproic acid (VPA).



Figure 2: Brain MRI. T2WI showed no abnormalities (TR $4000 \mathrm{msec}$, TE $98.2 \mathrm{msec}$ ). 
Citation: Saito T, Harahap ISK, Fujimura H, Saito K, Nishio H (2017) Valproic Acid Treatment for Spinal Muscular Atrophy Patient with Abnormal Electroencephalogram Findings. J Neurol Disord 5: 367. doi: 10.4172/2329-6895.1000367
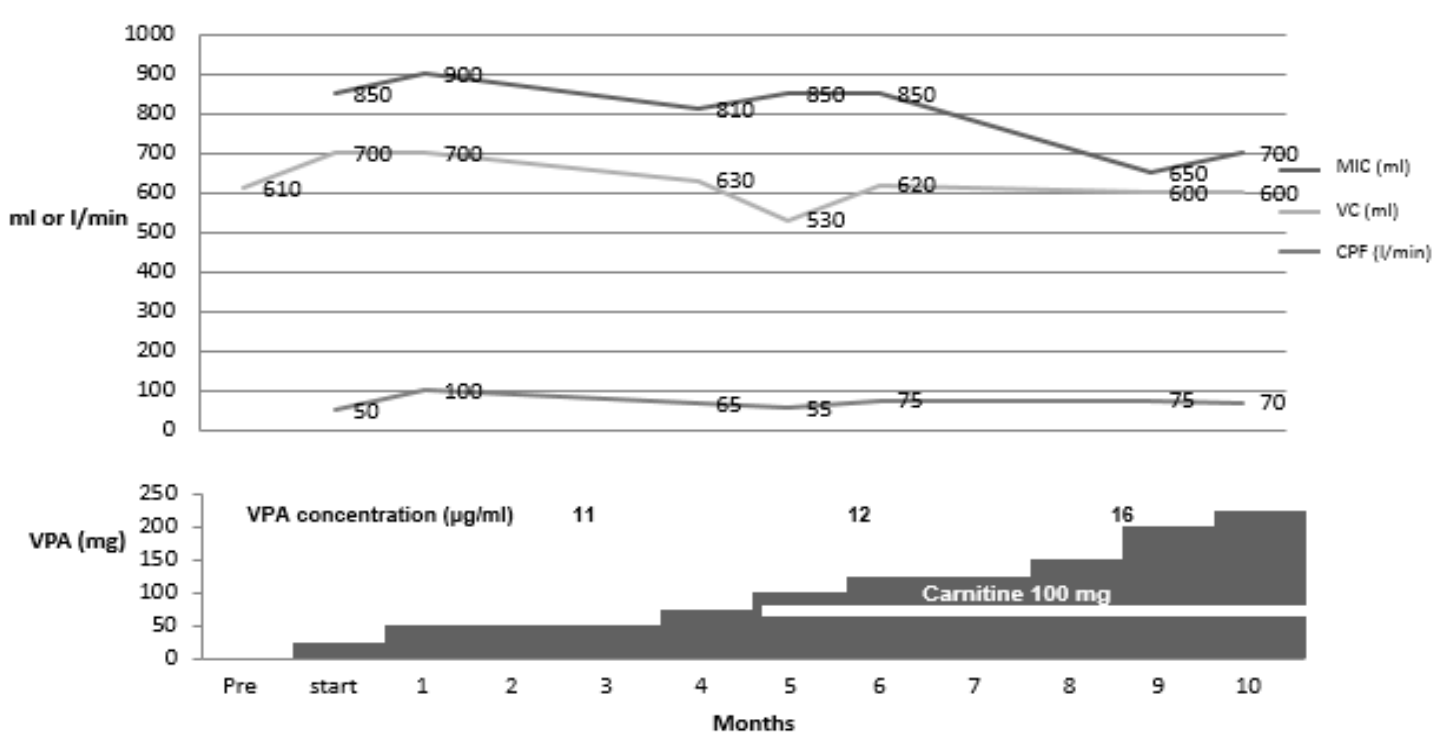

Figure 3: Sequential changes in respiratory function, VPA administration, and serum concentrations of VPA. We assessed vital capacity (VC), maximum insufflation capacity (MIC), and cough peak flow (CPF).

A FL-SMN

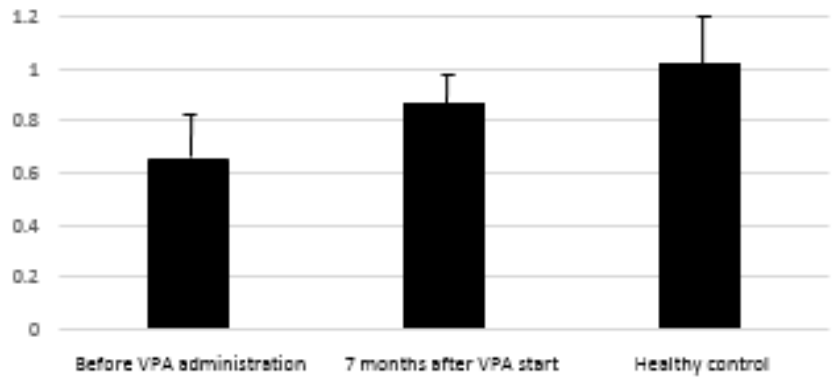

B $\triangle 7-S M N$
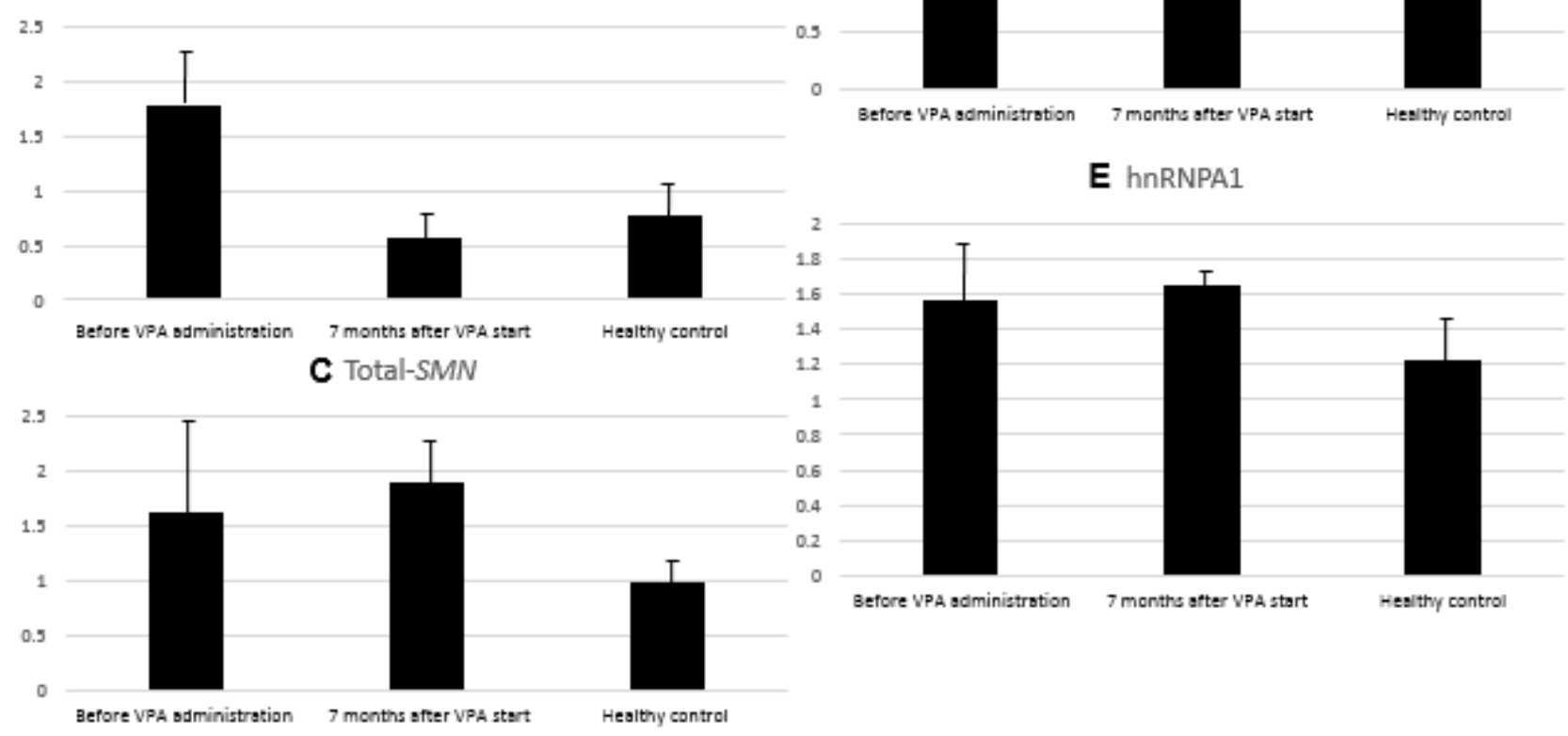

Figure 4: A: FL-SMN, B: $\triangle 7-S M N$, C: total-SMN, D: SF2/ASF, E: hnRNPA1. The transcript amounts of FL-SMN, total-SMN, SF2/ASF, and hnRNPA1 showed an


nuclear ribonucleoprotein A1 (hnRNPA1). 
Citation: Saito T, Harahap ISK, Fujimura H, Saito K, Nishio H (2017) Valproic Acid Treatment for Spinal Muscular Atrophy Patient with Abnormal Electroencephalogram Findings. J Neurol Disord 5: 367. doi: 10.4172/2329-6895.1000367

Page 4 of 4

and respiratory functions. On the other hand, the transcription amounts of FL-SMN, SF2/ASF, and hnRNPA1 showed an increasing tendency, which was considered to be an effect of VPA, even at a low concentration.

There are some limitations in regard to this study. First, the VPA concentration in our patient was in a low range, 50 to $100 \mu \mathrm{g} / \mathrm{ml}$, which is considered to be moderate for epilepsy treatment. Although VPA concentration does not necessarily correlate with motor function improvement of MHFMS [12], that in our patient might have been too low to have effects on motor and respiratory functions. In addition, we cannot exclude the possibility that changes in SMN, SF2/ASF, and hnRNPA1 transcript amounts in peripheral blood leukocytes may not necessarily correlate with changes in motor neuron functions. VPA, which is an HDAC inhibitor, might cause changes in the SMN, SF2/ ASF, and hnRNPA1 transcripts in motor neurons in the present case. Even the low concentration of VPA in the present case had effects on the central nervous system, shown by the resolution of electroencephalogram findings.

Finally, subtle changes in clinical signs and symptoms, such as MHFMS and respiratory function, may not have been detected by the evaluation methods employed. In our previous study of VPA therapy for SMA, we found motor function improvement in a child and improvements of respiratory functions in elderly patients who received the drug [12]. However, the efficacy of VPA therapy for SMA patients is controversial, as not all show a response. Appropriate methods to evaluate its efficacy in those patients more precisely are needed. Previous reports have described electroencephalogram abnormalities in SMA patients. The form of SMA has been reported to be related to the ASAH1 gene and the disease is known to be accompanied by progressive myoclonic epilepsy (SMA associated with progressive myoclonic epilepsy; SMA-PME) [15]. Although our patient showed electroencephalogram abnormalities, no epileptic attack was observed. Thus, we did not evaluate the ASAH1 gene in the present case, as the details did not match ASAH1-related SMA.

\section{Conclusion}

In conclusion, our findings indicate a discrepancy between response of $S M N$ transcripts to VPA, and improvements in signs and symptoms in some SMA patients.

\section{Acknowledgments}

The present findings were reported at the $49^{\text {th }}$ Kinki District Meeting of the Japanese Society of Child Neurology (Nara, Japan).

\section{Authors' Contributions}

All of the authors significantly contributed to production of this report. TS was the principal investigator, ISKH evaluated and analyzed the SMN transcript amounts, TS wrote the first draft of the paper, and HF, KS, and HN reviewed the drafts of the paper. All authors interpreted data, contributed to subsequent versions of the text, and approved the final version of the manuscript.

\section{Funding}

This work was supported by a Grant-in-Aid from the Ministry of Education, Science, Sports and Culture of Japan, and a Grant-in-Aid from the Research Committee of Spinal Muscular Atrophy (SMA) from the Ministry of Health, Labour and Welfare of Japan (Project number 25860860). Support was also received from the Practical Research Project for Rare/Intractable Diseases of the Japan Agency for Medical Research and Development (AMED) (Grant Number 16ek0109086h0002; title: "Practical study for multicenter cooperative and investigator initiated clinical trial using valproic acid in childhood onset spinal muscular atrophy").

\section{References}

1. Kolb SJ, Kissel JT (2011) Spinal muscular atrophy: A timely review. Arch Neurol 68: 979-984.

2. Lefebvre S, Bürglen L, Reboullet $S$, Clermont $O$, Burlet $P$, et al. (1995) Identification and characterization of a spinal muscular atrophy-determining gene. Cell 80: 155-165

3. Lefebvre S, Burlet $\mathrm{P}$, Liu Q, Bertrandy S, Clermont O, et al. (1997) Correlation between severity and SMN protein level in spinal muscular atrophy. Nat Genet 16: $265-269$.

4. Monani UR, Lorson CL, Parsons DW (1999) A single nucleotide difference that alters splicing patterns distinguishes the SMA gene SMN1 from the copy gene SMN2. Hum Mol Genet 8: 1177-1183.

5. Cartegni L, Krainer AR (2002) Disruption of an SF2/ASF-dependent exonic splicing enhancer in SMN2 causes spinal muscular atrophy in the absence of SMN1. Nat Genet 30: 377-384.

6. Kashima T, Manley JL (2003) A negative element in SMN2 exon 7 inhibits splicing in spinal muscular atrophy. Nat Genet 34: 460-463.

7. Harada $\mathrm{Y}$, Sutomo R, Sadewa AH, Akutsu T, Takeshima $\mathrm{Y}$, et al. (2002) Correlation between SMN2 copy number and clinical phenotype of spinal muscular atrophy: Three SMN2 copies fail to rescue some patients from the disease severity. J Neurol 249: 1211-1219.

8. Brichta L, Hofmann Y, Hahnen E (2003) Valproic acid increases the SMN2 protein level: A well-known drug as a potential therapy for spinal muscular atrophy. Hum Mol Genet 12: 2481-2489.

9. Sumner CJ, Huynh TN, Markowitz JA, Perhac S, Hill B et al. (2003) Valproic acid increases SMN levels in spinal muscular atrophy patient cells. Ann Neurol 54: 647-654

10. Swoboda KJ, Scott CB, Reyna SP, Prior TW, LaSalle B, et al. (2009) Phase II open label study of valproic acid in spinal muscular atrophy. PLoS One 4: e5268.

11. Swoboda KJ, Scott CB, Crawford TO, Simard LR, Reyna SP, et al. (2010) SMA CARNI-VAL trial part I: Double-blind, randomized, placebo-controlled trial of L-carnitine and valproic acid in spinal muscular atrophy. PLoS One 5: e12140.

12. Saito T, Nurputra DK, Harahap NI, Harahap IS, Yamamoto H, et al. (2015) A study of valproic acid for patients with spinal muscular atrophy. Neurol Clin Neurosci 3: 49-57.

13. Kang SW, Bach JR (2000) Maximum insufflation capacity: Vital capacity and cough flows in neuromuscular disease. Am J Phys Med Rehabil 79: 222-227.

14. Harahap IS, Saito T, San LP, Sasaki N, Gunadi, et al. (2012) Valproic acid increases SMN2 expression and modulates SF2/ASF and hnRNPA1 expression in SMA fibroblast cell lines. Brain Dev 34: 213-222.

15. Zhou J, Tawk M, Tiziano FD, Veillet J, Bayes M, et al. (2012) Spinal muscular atrophy associated with progressive myoclonic epilepsy is caused by mutations in ASAH1. Am J Hum Genet 91:5-14. 